\title{
Ehrlich's Methylenblaumethode und ihre Anwendung auf das Auge.
}

Von

Dr. Friedr. Hosch in Basel.

(Mittheilung aus dem normal-anatomischen Institut in Basel.)

Hierzu Ta敌l I-II, Fig. 1-8.

Zur Darstellung der peripheren Nervenenden stand uns bis vor Kurzem eigentlich nur die im Jahre 1866 von Cohnheim eingeführte Vergoldungsmethode zu Gebote. Wenn dieselbe seither auch wesentlich verbessert und vervollkommnet worden ist -- ich erwähne nur die Namen Ranvier und Löwitt -, so wird doch Jeder, der sich etwas eingehender damit beschäftigt hat, zugeben müssen, dass trotzdem diese Methode von capriciosester Natur ist, recht oft fehlschlägt und noch häufiger unerklärliche Artefakte liefert.

Wir haben daher allen Grund, Professor Ehrlich in Berlin dankbar zu sein, dass ex uns im Jahre 1886 in der Deutschen medic. Wochenschrift ein neues Verfahren die Nervenendigungen zu färben, gelehrt hat, das bei einiger Technik stets gelingt und das namentlich nicht, wie die Goldmethode, nebenbei noch alle möglichen Dinge mit tingirt. Auf gewisse Uebelstände, die leider auch diesem Verfahren anhaften, an deren Vermeidung aber, wie ich gleich bemerken will, schon mit grossem Erfolg gearbeitet worden, 
werde ich später zu reden kommen. Da die Methode in unserer Fachliteratur meines Wissens noch gar nicht Erwähnung gefunden hat, so will ich erst die Technik, wie sie ursprünglich ron Ehrlich empfohlen und seither recht glïcklich ausgebildet worden ist, etwas eingehender schildern.

Wenn man einem lebenden Frosch oder einem frisch getödteten Säugethier oder Vogel (bei letzteren, überbaupt Warmblütern, wirkt Methylenblau als heftiges, rasch tödtendes Gift) eine gesättigte Lösung von Methylenblau (in physiologischer Kochsalzlösung) in das Gefässsystem injicirt, so färben sich zunächst sämmtliche Organe, deren Gefässe vom Farbstoff erreicht werden, intensiv blau. Bald aber beginnen die gefärbten Theile blässer zu werden und haben nach kurzer Zeit, oft schon nach wenigen Minuten, jede Spur einer Blaufärbung wieder verloren.

Bringt man nun ein Stiickchen eines Organs, das vorher gefärbt war, unter das Mikroskop, so beobachtet man, wie unter dem Kinflusse der Luft (es darf also kein Deckglas aufgelegt werden) die letzten Spuren von Farbstoff die Gefässe verlassen, und mit ganz allmäliger Steigerung eine blaue Tinction der umgebenden Nervenelemente eintritt, „zuerst der Nervenfibrillen, der Nervenendapparate, der Nervenzellenfortsätze und der Zellen selbst, darauf der nackten Achsencylinder und marklosen Nervenfasern, der Ranvier'schen Kreuze und der Theilungsstellen der markbaltigen Fasern. Am schwierigsten färben sich die markhaltigen Nervenfasern, wahrscheinlich weil die Markscheide dem Methylenblau den Zutritt zu den Achsencylindern erschwert" (Dogiel).

Der Anblick ist für den, dem er zum ersten Male zu Theil wird, im höchsten Grade überraschend, und man begreift wohl, dass ein Mann wie G. Retzius, die Ehrlichsche Erfindung für „eine der bedeutungsvollsten Errungenschaften der neuesten histologischen Technik, wenn nicht geradezu für die vornehmste derselben" erklärt. 
Ehrlich's Methylenblaumethode und ihre Anwendung a. d. Auge. 39

Es mag hier am Platze sein, einige Worte über den Chemismus, der bei dem beschriebenen Vorgang in Action tritt, oder wenigstens wie derselbe von dem Erfinder aufgefasst wird, zu sagen.

Zunächst konnte Ehrlich durch mühsame Controllversuche sich davon überzeugen, dass das salzsaure Methylenblau, als dessen Formel

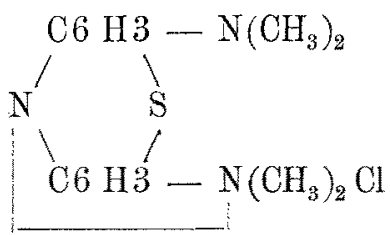

anzusehen ist, seine nervenfärbende Eigenschaft dem Fintritt des Schwefels in dieselbe verdankt und dass sie mit Elimination des letzteren dahinfällt.

Von Seite der Gewebe miissen nach der Ansicht von Ehrlich zum Gelingen der Nervenfärbung drei Bedingungen erfullt sein:

1) müssen dieselben gefässhaltig sein;

2) müssen die betreffenden Nervenfasern mit Sauerstoff gesättigt sein;

3) müssen sie alkalisch reagiren.

Sein Schüler Aronsohn führt dies in seiner Dissertation weiter aus: „Das Methylenblau ist ein sogen. küpenbildender Farbstoff. Durch reducirende (resp. Sauerstoff entziehende) Agentien wird er verhältnissmässig leicht, indem zwei H-Atome aufgenommen werden, zu Leukomethylenblau reducirt, welcher Körper sich bei Luftzutritt wieder von selbst in sein blates Oxydationsproduct verwandelt. Während des Lebens sind die Nerven so gut mit 0 versorgt, dass sie das von ihnen aufgenommene Methylenblau nicht zu reduciren vermögen. Nach dem Tode des Thieres werden dieselben wie fast alle iibrigen blau gefärbten Ele- 
mente farblos, d. h. nachdem die Zufuhr der natürlichen Sauerstoffspender aufgehört hat, wachsen die Sauerstoff anziehenden Affinitäten des Protoplasma derart, dass sie den 0 jetzt dem Methylenblau zu entziehen im Stande sind. Jedoch nehmen die farblosen Gewebe, in specie die Nerven, wenn sie - zumal in dünnen Schichten - der Luft ausgesetzt werden, ihre ursprüngliche blaue Farbe wieder an."

Dieser Process der secundären Oxydation, wie Aronsohn den beschriebenen Vorgang nennt, wird nun von anderen Beobachtern (Schwalbe, Feist) in Abrede gestellt. Sie wollen nichts davon wissen, dass die betreffenden Nerven schon einmal gefärbt gewesen seien und durch Mangel an 0 schon intra vitam oder erst beim Tode inre Farbo wieder verloren hätten, sondern nehmen einfach an, dass die Blautinction unter dem Einflusse des atmosphärischen Sauerstoffs, zusammen mit gewissen chemischen oder physilkalischen Veränderungen, welche die Gewebe beim Absterben erleiden, eintritt.

Ich will mich bei diesem noch streitigen Punkte nicht länger aufhalten. Da ich nur am todten Thier experimenlirt habe, kann ich mir ein bestimmtes Urtheil in dieser Hinsicht nicht erlauben.

Wie Dogiel gezeigt hat, kann man ein ähnliches Resultat, wie durch Infusion des blauen Farbstoffes in das Blutgefässsystem, auch erreichen, wenn man die zu färbenden Gewebe auf dem Objectträger mit rerdünnter Methylenblaulösung behandelt, bei freiem Zutritt der Luft. Unter seinen Augen sieht man bald, schon nach 5-10 Minuten, die vorhandenen Nerven sich färben, anfangs sehr schwach, allmälig aber wird die Färbung eine stärkere, bis nach Verlauf einer gewissen Zeit, alle eingelagerten Nervenelemente in der gleichen Reihenfolge wie bei der Injection tingirt sind. Allerdings färben sich hierbei, im Gegensatz zu letzterer, noch allerlei Zellen und bilden sich etwas störende Niederschläge, so dass sich das Resultat doch nicht 
ganz dem bei der Injection erhaltenen an die Seite stellen lässt.

Wie bereits angedeutet, hat auch diese Methode, so trefflich sie sonst ist, gewisse Uebelstände und Mängel an sich. Einer der unangenehmsten ist, dass die Färbung sehr vergänglich ist. Nach kurzer Zeit, oft schon nach $10 \mathrm{Mi}-$ nuten, beginnen die tingirten Theile abzublassen, und sehr bald ist von dem so schönen Bilde nichts mehr übrig geblieben; ein hellblauer Saum umrandet noch das Präparat, und auch dieser ist binnen Kurzem verschwunden.

Es ist sehr begreiflich, dass man alle Anstrengungen gemacht hat, die mit solcher Mühe erhaltenen Präparate zu retten oder die Färbung derselben wenigstens so lange zu fixiren, dass sie gezeichnet und genügend durchstudirt werden kömnen. In diesem Bestreben haben sich namentlich Prof. Arnstein in Kasan und seine Schüler Smirnow und Dogiel, grosse Verdienste erworben. Zunächst wurde hierzu eine eimprocentwässerige Lösung von Jodkalium verwendet, in welcher metallisches Jod bis zur Sättigung gelöst ist. Mit dieser Lösung wird am besten das Blutgefässsystem durchspült. Dann werden die Gewebsstücke ausgeschnitten, 6-12 Stunden in die Jodlösung gelegt und ausgewässert. Jetzt heben sich die früher blassen Nerven in schwarzbrauner oder grauer Farbe auf dem fast farblosen Grunde ab. Noch bessere Resultate erhielt dann Smirnow mit einer Lösung von Hoyer'schem Pikrokarmin, als dessen fixirendes Agens von Dogiel das pikrinsaure Ammoniak erkannt wurde. Seither wird wohl nur noch das letztere in concentrirter wässeriger Lösung zur Fixirung benützt und giebt recht schöne Resultate. Das Methylenblau wird in Form eines feinkörnigen, violetten Niederschlags gefällt, zugleich aber das Grundgewebe durchsichtig gemacht und somit ermöglicht, auch relativ dicke Häutchen und Gewebsstücke noch in toto zu untersuchen. Zudem wird durch das pikrinsaure Ammoniak das Gewebe 
gelockert and zum Zexzupfen sehr geeignet. Ich habe alle mir bekannt gewordenen Fixirmittel durchprobirt, bin aber stets gerno wieder zum pikrinsauren Ammoniak zurückgekebrt.

Weniger glücklich ist man bis jetzt gewesen mit den Versuchen, die Methylenblaupräparate zu härten oder sonst wie zum Schneiden geeignet zu machen. Nach meinen schmerzlichen Erfahrungen zieht schon die geringste Spur ron Alkohol, Aether oder ätherischen Oelen den Farbstoff in rapidester Weise aus. Auch Versuche, die Präparate in Klebs'schen Glycerinleim einzuschliessen, sind mir bis dahin total missglückt, da dieselben die zum Flüssigmachen des Leims nöthige Temperatur absolut nicht ertrugen. Ebensowenig führte mich der von Feist empfohlene Einschluss in Gummiglycerin (nach Joliet) zu einem nemnenswerthen Resultate. Entweder war die Einschlussmasse zu weich und erlaubte dann nur sehr dicke Schnitte, oder sie wurde rasch so hart, dass sie gar nicht mehr geschnitten werden konnte. Ein grosser Uebelstand ist dabei auch, dass man das Messer stark mit Glycerin befeuchten muss. Dass auch Andere beim Schneiden auf ganz besondere Schwierigkeiten gestossen sind, beweist mir die Thatsache, dass alle Empfehlungen in dieser Hinsicht durchweg höchst ungenau redigirt sind. Meiner Ansicht nach verdient einstweilen nur das Schneiden der frisch gefärbten Präparate mit dem Gefriermikrotom und nachheriges Fixiren mit pikrinsaurem Ammoniak wirkliches Vertrauen. Leider bekommt man dabei aber wohl nur ziemlich dicke Schnitte, die das Erkennen feinerer Einzelnheiten und die Anwendung stärkerer Vergrösserungen nicht zulassen.

Ich habe nun, auf Anregung von Herrn Prof. Kollman, welchem ich für seine Unterstützung hiermit bestens danke, es unternommen zu untersuchen, welche Resultate die so viel versprechende neue Methode am Ange zu Tage zu fördern im Stande sei. Die Versuche wurden ausschliess- 
Ehrlich's Methylenblaumethode und ihre Anwendung a. d. Auge. 43

lich am albinotischen Kaninchen vorgenommen und bezogen sich in erster Linie auf die Cornea und Iris. Die Retina, welche schon von Dogiel selbst eine eingehende Bearbeitung erfahren hat, wurde nur nebenbei berücksichtigt.

Bei der Injection des Farbstoffes in das Blutgefässsystem verfuhr ich folgendermaassen: Zunächst wird das Thier zu Tode chloroformirt, dam rasch die Bauchhöhle eröffnet und eines der grossen Gefässe in derselben - zur möglichsten Entleerung des Circulationsapparates - durchschnitten. Nun werden Aorta ascendens und Art. pulmonalis zusammen unmittelbar über dem Herzen umschnürt und die Canüle mit der - kopfwärts gerichteten - Spitze in einen Schlitz der Aorta thoracica eingebunden.

Anfangs machte ich die Injectionen mit einer gewöhnlichen Injectionsspritze und kalter gesättigter Methylenblaulösung, kam aber nur ausnahmsweise und nur zum Theil zum gewünschten Ziele. Gewöhnlich stiess der Kolben der Spritze schon auf starken Widerstand, noch ehe die Blaufärbung der Obren und des übrigen Kopfes genügend eingetreten war. Wurde dann die Injection forcirt, so zeigte bald die da und dort austretende Farblösung an, dass unliebsame Rupturen eingetreten seien und damit das Experiment fehlgeschlagen habe.

Da Herr Prof. Kollmanu der Ansicht war, dass an diesem Missgeschick nur ein hochgradiger Gefässkrampf Schuld sein könne, so habe ich mir mittelst Trichter und Gummischlauch einen ganz einfachen Injectionsapparat für constanten Druck zusammengestellt und die Iujectionsfliussigkeit jeweilen im Wasserbade auf Bluttemperatur erwärmt. Damit waren jene Uebelstände sofort beseitigt, und die Injectionen geschehen jetzt mit einer Sicherheit und Schnelligkeit, wie man sie nicht besser wünschen kann. Schon nach Abfluss ron wenigen Cubikcentimetern der Farblösung färben sich die Ohren intensiv blau, bald auch die Schnauze und der übrige Kopf. Nach wenigen Minuten ist die Con- 
junctiva und bald auch die Iris dunirelblau gefärbt. In letzterer treten zuerst vier Gefässe in Form yon dicken blauen Strängen auf, die aber unter der allgemeinen Bläuung in kurzer Zeit wieder verschwinden.

Nach meiner Erfahrung ist es am Platze, damit auch die feinen Gefässe sich füllen und die Färbung der Nerven bis in die Peripherie genügend sei, mit der Infusion noeh etwas fortzufahren. Allerdings werden auf diese Weise die an Gefässen besonders reichen Theile, wie Chorioidea und Ciliarkörper, wegen der reichlichen Farbstoffextravasate, für die spätere Untersuchung nahezu unbrauchbar. Dafür hat man aber, wie erwähnt, den Vortheil, dass die Färbung der für den gewünschten Zweck wichtigen Parthieen eine um so intensivere ist und bleibt.

Die Dauer der Injectionen überschreitet selten fünf Minuten, seit ich dieselben in der beschriebenen Weise vorzunehmen pflege.

Nach kurzer Zeit blassen die blau gefärbten Theile wieder ab. Nur an den der Luft ausgesetzten Stellen, wie im Bereich der Lidspalte, bleibt die Färbung länger bestehen.

Alle Beobachter geben den Rath, das injicirte Thier eine Zeit lang liegen zu lassen und erst dann die zu untersuchenden Gewebsstücke auszuschneiden und - unter freiem Zutritt der Luft, also ohne Deckglas - auf den Objectträger zu bringen. Wie lange man warten soll, darüber finden sich aber fast überall andere Angaben, welche zwischen $1 / 4$ und drei, sogar noch mehr Stunden schwanken.

Ioh habe nun aus meinen Versuchen den Eindruck gewonnen, als könne, vorausgesetzt, dass die Färbung eine genügende war, die Untersuchung vorgenommen werden, sobald die Abblassung eingetreten ist und damit der Farbstoff die Blutgetässe verlassen hat. Aehnlich wie bei den Objectträgerfärbungen sehen wir jetzt, unter dem Einflusse des atmosphärischen Sauerstoffs, vor unseren Augen die 
Ehrlich's Methylenblaumethode and ibre Anwendung a. d. Auge. 45

feinsten Ausbreitungen des Achsencylinders ganz allmälig sich blau färben und können ganz sicher den Zeitpunkt bestimmen, von welchem an die Färbung nicht mehr weiter schreiten, sondern zurückgehen wird. Dies ist der Moment, wo es sich darum handelt, dem so vergänglichen Bilde Dauer zu geben, resp. dasselbe zu fixiren.

Es ist dies ohne Zweifel der heikelste Theil des ganzen Versuchs, was schon aus den zum Theil sehr unbestimmten, zum Theil sehr differenten Angaben der Autoren hierüber $\mathrm{zu}$ ersehen ist. Ich habe oben erwähnt, dass ich nach mehrfachen Proben mit anderen Fixirmitteln stets wieder zur gesättigten Lösung des pikrinsauren Ammoniaks zurückgekehrt bin. Ueber die Dauer seiner Einwirkuug bestimmte Regeln aufstellen zu wollen, scheint mir ganz unstatthaft; sie ist eben für jeden einzelnen Fall wieder eine andere, ohne dass sich eine bestimmte Erklärung dafür auffinden liesse. Nach einiger Uebung, aber auch nach mancher Enttäuschung bringt man es jedoch dahin, mit ziemlicher Sicherheit den Moment zu bestimmen, in welchem die Fixation eine genügende ist und unterbrochen werden darf. Durch das pikrinsaure Ammoniak wird das Methylenblau in Form eines feinkörnigen violetten Niederschlags gefällt; doch bedarf dies an verschiedenen Stellen des gleichen Präparates einer verschieden langen Einwirkung desselben. Man hat also einfach abzuwarten, bis alles, was vorher blau war, entschieden violett geworden ist. Auf diese Weise habe ich Präparate bekommen, die nun schon 5-6 Monate alt sind und alle Details noch so vollkommen zeigen ${ }^{1}$, wie unmittelbar nach der Fixirung, während andere, bei denen ich die Fixirung $\mathrm{zu}$ früh unterbrochen, allmälig vollständig abgeblasst sind.

I) Leider bin ich gezwungen, die obige Angabe, aus der man schliessen könnte, die Präparate hätten nunmehr eine unbegrenzte Haltbarkeit gewonnen, etwas zu modificiren. Die alltesten derselben, welche aus der ersten Hälfte des November 1890 stammen, und noch 
Die fxirten Präparate werden am besten in Glycerin aufbewahrt. Einige Autoren empfehlen diesem etwas pikrinsaures Ammoniak zuzusetzen. Mir scheinen die - gut fixirten - Präparate ohne solches hübscher und ebenso haltbar zu sein. Um diejenigen Präparate, welche aufbewahrt werden sollen, pllege ich einen Rand von Klebs'schen Glycerinleim zu ziehen.

Was zunächst die Cornea anbelangt, so sieht man durch das Methylenblau sofort viele Zellen deutlich gefärbt. Ich hebe diesen Umstand besonders herror, weil er zeigt, dass nicht bloss Nervenfasem und Nervenzellen gefärbt werden. Es ist wahrscheinlich das specifische Protoplasma dieser Zellen, das ähnlich wie die Achsencylinder reagirt. Nach der Fixirung mit pikrinsaurem Ammoniak werden noch mehr Hornhautzellen, vielleicht alle, deutich. Hiernach wäre also eine Angabe Arnstein's zu modificiren, der an den Zellen vor der Fixirung keine Färbung wahrnahm.

Die Grundsubstanz der Cornea bleibt nach- wie vorber ungefärbt, wodurch das Präparat äusserst durchsichtig wird und sämmtliche Nervenverzweigungen sich deutlichst verfolgen lassen, zum Unterschiede von der Goldbehandlung, bei der auch das Grundgewebe stark sich färbt, weshalb sie für Flächenpräparate nur bei dünnen Hornhäuten oder bei Zerzupfung in Lamellen sich eignet.

Die mit Methylenblau erhaltenen Flächenpräparate stimmen ziemlich genau mit der Beschreibung, welche Ranvier und Schwalbe geben. Die am Rande eintretenden, sofort marlslos werdenden, aus dicht gedrängten feinen und mit zarten Varicositäten versehenen Fibrillen bestehenden Nerven verlaufen, nach Art der Aeste eines Baumes wiederholt

am 30. Mai d. J. der schweizerischen Aerzte-Versammlung in ihrer ganzen Schönheit gezeigt werden konnten, beginnen nun auch in ihren zartesten Parthieen abzublassen und werden wohl bald ganz unbrauchbar geworden sein. 
Ehrlich's Methylenblaumethode und ihre Anwendung a. d. Auge. 47

dichotomisch sich theilend und reichlich unter einander anastomosirend, gegen die Hornhautmitte hin, um dort den Basalplexus zu bilden. An den Theilungsstellen kommt jeweilen eine Verbreiterung zu Stande; die bisher dicht aneinander liegenden Axencylinderfibrillen weichen auseinander und durchkreuzen sich auf das Mannigfachste nach allen Richtungen hin. In diese Knotenpunkte sind fast regelmässig längliche Kerne eingelagert, welchen $\mathrm{His}$ die Bedeutung von Ganglienzellen beilegte, während Hoyer u. A. m. sie - wohl mit Recht - nur als Bestandtheile der die Fibrillen vereinigenden Neuroglia betrachten (Figur 1).

Nach dem Durchtritt durch den Knoten sammeln sich die Fibrillen zu entsprechend verjüngten Stämmchen, indem sie sich innigst aneinander lagern, um beim nächsten Knotenpunkte wieder in gleicher Weise auseinander zu gehen und sich von Neuem zu durchflechten. Dieser Vorgang wiederholt sich mehrmals, während das Nervenästchen in radiärer Richtung der Hormhautmitte zustrebt und auf diesem Wege sowohl mit den benachbarten Zweigen als auch mit den zwischen den vorderen Schichten der Hornhaut eintretenden Aestchen Verbindungen eingeht.

Die Anastomosen, welche die einzelnen Nervenstämmchen unter sich bilden, sind sehr verschieden, bald etwa steif, ähnlich den Zweigen eines Baumes, bald feine, leicht gebogene Bälkchen bildend. Ein sehr zierliches Bild ergiebt sich, wenn ein solches Bälkchen spiralig um ein dickeres Aestchen sich herumwindet. Nicht selten wird die Verbindung auch durch eine einzige Fibrille besorgt, und zwar meist nicht auf dem kürzesten Wege, sondern die Faser strebt erst in einem Bogen gegen die Hornhautmitte, um dann ganz plötzlich unter einem fast rechten Winkel gegen die Anastomose sich zu wenden. So entstehen die zierlichsten und mannigfaltigsten Bilder, die um so mehr frappiren, weil sie sich so deutlich von dem farblosen Grunde abheben.- Fig. 2 mag einen ungefähren Begriff geben von 
der Mannigfaltigkeit und dem Reichtbum der in einem einzigen Gesichtsfelde enthaltenen Nervenverzweigungen.

In einem Präparate fand sich eine besonders auffallende Anastomosenbildung vor; unmittelbar aus einem Knoten tritt ein stark gekörnter Strang hervor, der nach kurzem Verlauf ganz plötzlich in eine olivenförmige, ebenfalls gekörnte und sehr dunkel gefärbte Anschwellung ausläuft. Aus dieser Anschwellung geht nun ein feiner Faden bervor, welcher in bogenförmigem Verlaufe mit einer ähnlichen olivenförmigen Anschwellung sich verbindet, die aus einem anderen Knoten des Grundplexus sich entwickelt (Fig. 3). $\mathrm{Ob}$ wir es hier mit einer normalen oder pathologischen Bildung - vielleicht den Resten einer früheren Verletzung - zu thun haben, konnte ich bis jetzt nicht eruiren. Auf letzteres scheint mir einigermaassen der Umstand zu deuten, dass diese Bildung sich eben nur in einer einzigen Cornea, wenn auch mehrfach, vorfand.

Einen Uebergang der Stromanerven in das Protoplasma der Hornhautzellen und Endigung in den Nerven derselben, wie es namentlich ron Lavdowski behauptet und auf das Deutlichste gezeichnet wird, vermochte ich ebenso wenig nachzuweisen als die meisten anderen Beobachter. Da, wo eine solche innigere Beziehung zu den Hornhautzellen rorhanden schien, konnte bei genauerer Untersuchung oder Anwendung stärkerer Vergrösserung jeweilen constatirt werden, dass die betreffenden Nervenfasern sich bloss den Rändern der Zellen oder ihrer Oberfläche anlegten oder über sie hinwegliefen.

Für diese Beobachtung eignen sich die so durchsichtigen Methylenblaupräparate ganz ausgezeichnet, während dagegen die Frage, ob die Nervenfasern zu ihrer Ausbreitung vorzugsweise die Saftkanälchen benutzen, wie behauptet wird, mittelst derselben sich kaum entscheiden lässt. Immerhin wird wohl Hoy er Recht haben, wenn er annimmt, dass sie mit Vorliebe diese vorgebildeten Wege wählen, 
Ehrlich's Methylenblaumethode und ihre Anwendung a. đ. Auge. 49

weil ihnen eben dort am wenigsten Hindernisse entgegentreten, gleichwie auch die Wanderzellen bei entzündlichen Zuständen vorzugsweise nach dem vom Protoplasma erfüllten Lückensystem sich zu drängen pllegen.

Vom Hauptgeflecht trennen sich mehr weniger lange Fäden, welche senkrecht oder schräg nach vorn verlaufen, als rami perforantes die Basalmembran durchbohren und unter dem vorderen Epithel den subepithelialen Plexus bilden. Von diesem sieht man wiederum nach allen Richtungen eine Unmasse von foinsten, mit Varicositäten versehenen Fibrillen abgehen, welche meist auf lange Strecken ganz geradlinig, zuweilen auch in verschiedenen Richtungen sich schlängelnd verlaufen, sich auch verzweigen und mit anderen Fibrillen kreuzen und verbinden. Geht man diesen feinen Fädchen bis ans Ende nach, wozu man nicht selten einen grossen Theil des Präparates durchsuchen muss, so findet man, dass sie mit kleinen Knöpfchen, ähnlich den im Verlaufe des Fadens vorhandenen varicösen Anschwellungen, zuweilen auch mit einer schaufelförmigen Verbreiterung - scheinbar frei - enden (Fig. 4).

Um hierüber ins Klare zu kommen, sind feine senkrechte Durchschnitte unerlässlich. Da nun aber die Methylenblaupräparate, wie mitgetheilt, hierfür nicht zu verwenden sind, habe ich Kaninchenhornhäute nach der Vorschrift Ranvier's mit Gold behandelt, in Alkohol gehärtet und in Paraffin eingebettet. Es ergab sich nun des Unzweifelhaftesten, dass die Fäden bis zu den oberflächlichen Pflasterzellen des Epithels hinaufsteigen und dort mit einer knopfförmigen Anschwellung enden (Fig. 5). Hoyer erklärt zwar sowohl diese knopfartigen Verdickungen an den Enden, als auch die Varicositäten im Verlaufe der Fibrillen sämmtlich für Kunstprodukte, welche theils durch unvollkommene und ungleichmässige, theils durch zu intensive Goldwirkung erzeugt sein sollen. An besonders gelungenen Präparaten, wie Fig. 5, sind die "Endknöpfchen", obschon sie keinerlei

v. Graefe's Archiv für Ophthalmologie. XXXVIT. 3. 
weitere Structur erkennen lassen, jedoch von so charakteristischem Aussehen und bilden so sehr das natürliche Ende der Nervenfaser, dass man sie nur mit Zwang für Artefakte halten würde. Ich meines Theils stehe nicht an, in ihnen die Endorgane der sensiblen Hornhautnerven zu erkennen. Damit ist wohl die jüngst von Brand (Arch. für. Augenheilk. XIX) aufgestellte Behauptung, dass die Rami perforantes die letzten Endigungen der Hornhautnerven seien, und dass bei keiner Thierspecies das Nervenendorgan „über das Hornhautstroma hinaus" rage, widerlegt.

Der Nervenreichthum ist an den Methylenblaupräparaten ohne Zweifel noch grösser als an den Goldpräparaten, so gross, dass, wenn man nicht so leicht die directe Beziehung der feinsten Fibrillen zu sicher als solche erkennbaren Nervenfasern nachzuweisen vermöchte, man an der nervösen Natur derselben oft zweifeln könnte. Wir müssen uns eben angewöhnen mit Hilfe der Ehrlich'schen Erfindung in den Geweben viel mehr Nervenfasern aufzufinden, als wir nach den bisher gebräuchlichen Methoden darin zu vermuthen gewohnt waren. Bei der Cornea kann uns übrigens die beschriebene reiche Vertheilung der Nervenverzweigungen nicht so sehr wandern, wenn wir an die physiologische Aufgabe dieser Membran denken, welche bei vollständiger Durchsichtigkeit eine möglichst ausgebildete Sensibilität, vielleicht sogar eine directe Reizbarkeit den Lichtstrahlen gegenüber verlangt.

Auch bei der Iris zeigt sich die Methode von grossem Vortheil gegenüber anderen. Da die Regenbogenhaut des albinotischen Kaninchens viel za dick ist, um in toto ausgebreitet gute Uebersichtsbilder über den ganzon Verlauf der Nerven bis zu ihren Endigungen zu geben, so muss man gewöhnlich durch feine Flächenschnitte oder Abreissen von einzelnen Fetzen sich Auskunft über deren Verbreitung zu verschaffen suchen. Bei der Methylenblaumethode, wo durch die Procedur der Fixirung das Grundgewebe, wie 
Ehrlich's Methylenblaumethode und ihre Anwendung a.d. Auge. 51

wir schon bei der Cornea gesehen haben, ganz durchsichtig wird, sehen wir sämmtliche Nervenverzweigungen in übersichtlichster Weise auf farblosem Grunde. Es fällt auch hier zunächst der ungeheure Nervenreichthum auf, wie er durch keine andere Methode auch nur annähernd dargestellt wird.

Zweifellos die beste Beschreibung der Nervenvertheilung in der Iris der Säugethiere, speciell des Kaninchens, verdanken wir Al. Meyer (Archiv für mikr. Anat. 1879, S. 324). Er unterscheidet motorische Nerven, die in der Gegend des Sphincter meist aus nackten Fibrillenbündeln bestehen; sensible Nerven, deren Endapparate an der vorderen Oberfläche der Iris ein engmaschiges Netz bilden; und endlich vasomotorische Nerven, welche in sämmtlichen Schichten der Iris verbreitet sind.

Unsere Methylenblaupräparate ergeben nun zunächst, dass die markhaltigen Nerven in der Ciliargegend zwei durch reichliche Anastomosen verbundene circuläre Plexus bilden, ron denen der eine etwas tiefer liegt, als der andere. Von diesen aus streben korkzieherartig gewundene markhaltige Nervenfasern in radiärer Richtung - eine innigere Beziehung zum Verlauf der Gefässe ist mir nicht aufgefallen - nach dem äusseren Sphincterrande zu, wo sie sich wiederum zu einem, aus arkadenförmigen Windungen bestehenden Ringgeflecht gruppiren. Erst von da gehen vorwiegend marklose Fasern in das Gewebe des Sphincter $\mathrm{ab}$, um hier ein überaus reiches Netz von feinen, punktirten Fäden zu bilden. Ob dieses das eigentliche Terminalorgan bildet, demnach als Endnetz aufzufassen ist, oder ob die feinen Fasern noch in einer intimeren Beziehung zu den glatten Muskelfasern und deren Kernen stehen, konnte ich an den hierfür nur allzudurchsichtigen Präparaten bis jetzt nicht herausfinden (Fig. 6).

Selbstrerständlich babe ich mein Augenmerk ganz besonders auf das Vorhandensein von Nervenzellen gerichtet. Verschiedene Beobachtungen deuten unzweideutig darauf 
hin, dass solche secundäre nerröse Centren als Vermittler zwischen Muskeln und Nerven in der Iris enthalten sein müssen. So namentlich die von Brown-Séquard gefundene Thatsache, dass die Pupille der ausgeschnittenen Iris noch durch Lichteinwirkung sich verengert; ferner der Umstand, dass sowohl Mydriatica als Myotica, local applicirt, auch nach Durchschneidung des Ganglionciliare (Hensen und Völckers) und sogar am enucleirten Auge (de Ruyter) ihre Wirkung nicht versagen.

Und doch ist heute die Frage, ob in der Regenbogenhaut des Säugethieres Ganglienzellen sich vorfinden oder nicht, noch eine vollständig offene. Während ältere Beobachter (Arnold, Faber) solche in der Iris gesehen zu haben meinen, stellen die meisten neueren Autoren (Pause, Iwanoff, Fürst, Meyer, Schwalbe) deren Vorkommen in Abrede. Nur in der Regenbogenbaut des Menschen beobachtete Al. Meyer an Zupfpräparaten Zellen, die in Bezug auf Grösse, Zahl der Fortsätze, körniges Protoplasma und bläschenförmigen Kern den Ganglienzellen sehr ähnlich sahen, jedoch keinen Zusammenhang mit Nervenfasem erkennen liessen. Das Fehlen von Ganglienzellen in der Iris aller anderen Thiere glaubt er aus der Thatsache erklären zu müssen, dass solche in die Stämme der Ciliarnerven vor ihrem Eintritt in die Iris eingeschaltet sind.

Ich finde nun in dem beschriebenen nervösen Netz der Sphineterzone und an der äusseren Grenze derselben da und dort spindelförmige oder dreieckige, Kern und Kernkörperchen enthaltende Zellen eingeschaltet, die zwar etwas klein sind $(12-15 \mu)$, aber meist ganz den Ganglienzellen entsprechen und lange, aus punktirten Linien zusammengesetzte Fortsätze aussenden. In einzelnen Fällen (Fig. 7) konnte ich auch ganz sicher einen Zusammenhang des einen oder andern Ausläufers mit markhaltigen Nervenfasern nachweisen. Ich stehe daher nicht an, diese Gebilde als die postulirten Nervenzellen aufzufassen. 
Ehrlich's Metbylenblaumethode und ihre Anwendung a. d. Auge. 53

Sehr schön ist an einzelnen stärker gefärbten Präparaten das über die ganze Vorderfläche der Iris ausgebreitete, aus feinsten, kernlosen, punktirten Fädchen bestehende Maschenwerk dargestellt, welches nach Alex. Meyer dem Endapparat der sensibeln Nerven entsprechen soll.

An den Arterien sieht man nicht selten die Muskelkerne der Media violett gefärbt und das Gefäss in Form einer unterbrochenen Spirale umgeben. Sehr zierlich ist das Bild namentlich an den Stellen, wo das Gefäss blutleer ist. Dort treten dann auch die feinen Fäden des umspinnenden Nervenplexus deutlicher hervor, in welchem das Gefäss wie in einem grobmaschigen Garne aufgehängt erscheint.

Ueber meine bisherigen Beobachtungen an der Retina kann jch sehr rasch hinweggehen. Es macht sich hier eben namentlich der Mangel an genügend feinen Durchschnitten im höchsten Grade fühlbar. An Flächenpräparaten findet man vor Allem sehr schön gefärbt die multipolaren Ganglienzellen mit ihren Ausläufern (Figur 8), welche sich zu einem sehr eleganten, flächenförmig ausgebreiteten Netzwerk feiner varicöser Fibrillen gruppiren. Auch die Elemente der Körnerschichten nehmen die Färbung an, während die eigentlichen Sehzellen, die Stäbchen und Zapfen, durch das Methylenblau nie tingirt zu werden scheinen.

Das ist in groben Zügen, was mir die Ehrlich'sche Methode bis jetzt am Kaninchenauge ergeben hat. Dieselbe ist - bei weiterer Vervollkommnung in der angedeuteten Richtung - ohne Zweifel dazu berufen, noch manches Räthsel auf dem Gebiete der Anatomie des Nerrensystems zu lösen. Jedenfalls aber dürfen wir sie heute schon als eine höchst willkommene Ergänzung zu den bisher gebräuchlichen, in mancher Hinsicht so unzuverlässigen Metallimprägnationen betrachten. 


\section{Figurenerklärung.}

\section{Tafel I-II.}

Fig. 1. Knotenpunkt aus dem Basalplexus mit dichotomischer Theilung, mit zwei Kernen (Leitz Obj. 7. Cam. luc. Vergr. ca. 600. Daneben dieselbe Stelle in 40 facher Vergröss.). Wegen der grösseren Deutlichkeit sind viele Fibrillen weggelassen. Man sieht eine grosse Anzahl derselben sich theilen und nach beiden Seiten hin einen Faden abgeben.

Fig. 2. Uebersicht der in einem einzigen Gesichtsfelde sichtbaren Nervenverzweigungen. Is eitz Oc. 3. Obj. 3. Tubus eingesehoben.

Fig. 3. Spindelförmige Verdickung an einer Anastomose zwischen zwei Nervenstämmchen dex Cornea. a) Leitz Obj. 3. Cam. luc. b) Leitz Obj. 7. Cam. luc.

Fig. 4. Knotenpunkt aus dem subepithelialen Plexus, von welchem zablreiche Fibrillen ausgehen, die sich zum Theil dichotomisch theilen, zum Theil ubber weite Strecken hinweglaufen und mit kleinen Knöpfchen enden. Leitz Obj. 3. Cam. luc.

Fig. 5. Knopfförmige Nervenendigung im vorderen Hornhautepithel (Endknöpfchen). Goldpräparat nach Ranvier. Leitz Obj. 7. Cam. Iuc.

Fig. 6. Uebersichtsbild über die Verbreitungsweise der motorischen Nerven in der Iris. Leitz Oc. 3. Obj. 3. Aus technischen Gründen musste eine grosse Anzahl der in Wirklichkeit vorhandenen Verzweigungen, namentlich im Sphinctertheil, weggelassen werden.

Fig. 7. Nervenzelle aus der Sphincterzone der Iris, mit dem einen ihrer Fortsătze in eine markhaltige Nervenfaser übergehend,

Fig. 8. Multipolare Ganglienzellen aus der Retina. Leitz Obj. 7. Cam. luc.

Sämmtliche Präparate sind, wo nichts Anderes angegeben, gewonnen durch Injection dex gesättigten Methylenblaulösung in das Blutgefässsystem und nachherige Fixirung mit pikrinsaurem Ammoniak. 
v. Graefés Archiv Bd.XXXVII. s.

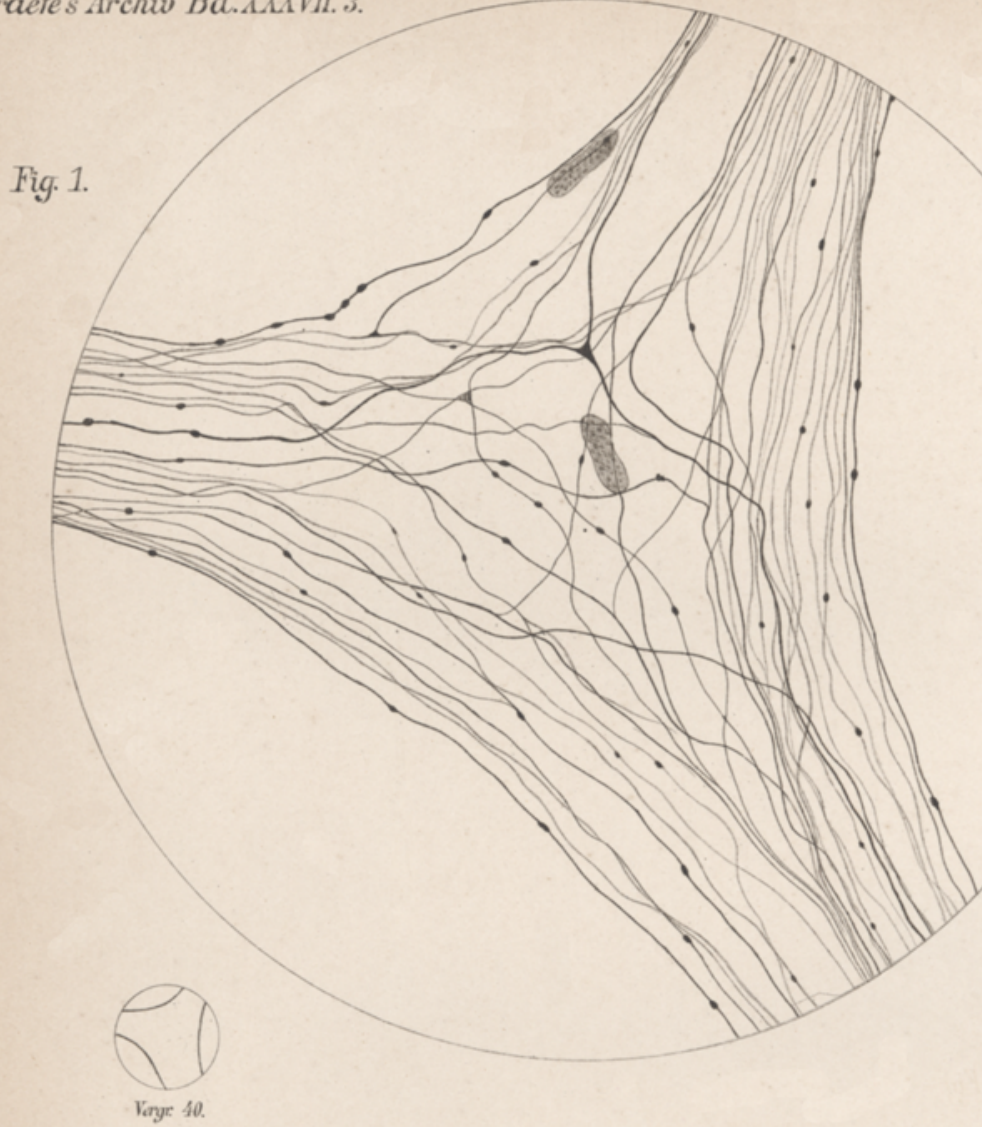

Fig. 3.

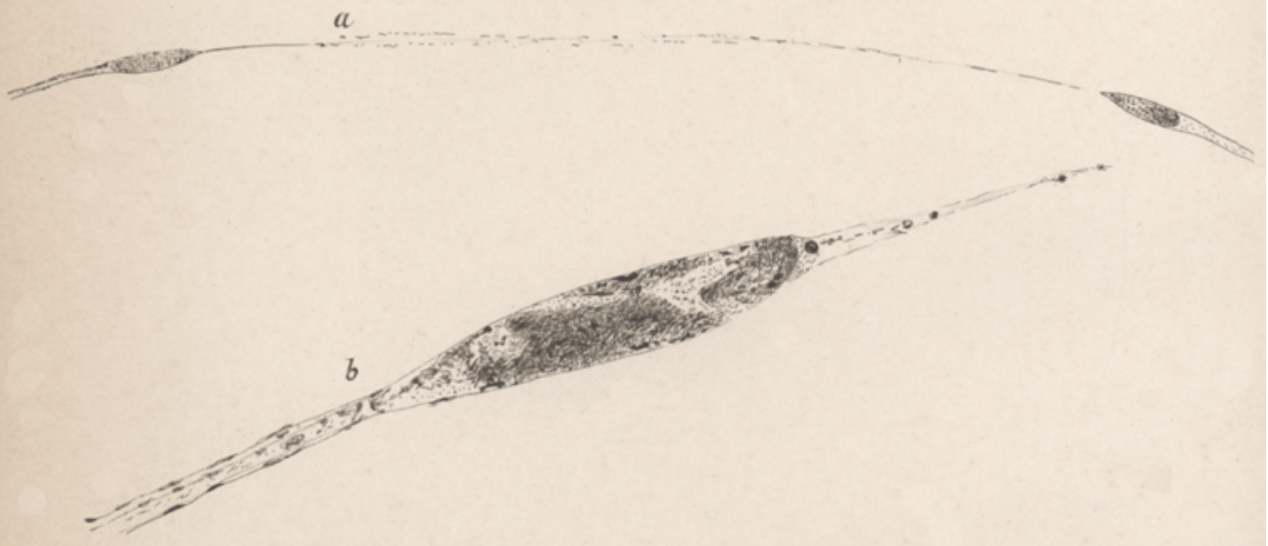


Iaf.I.

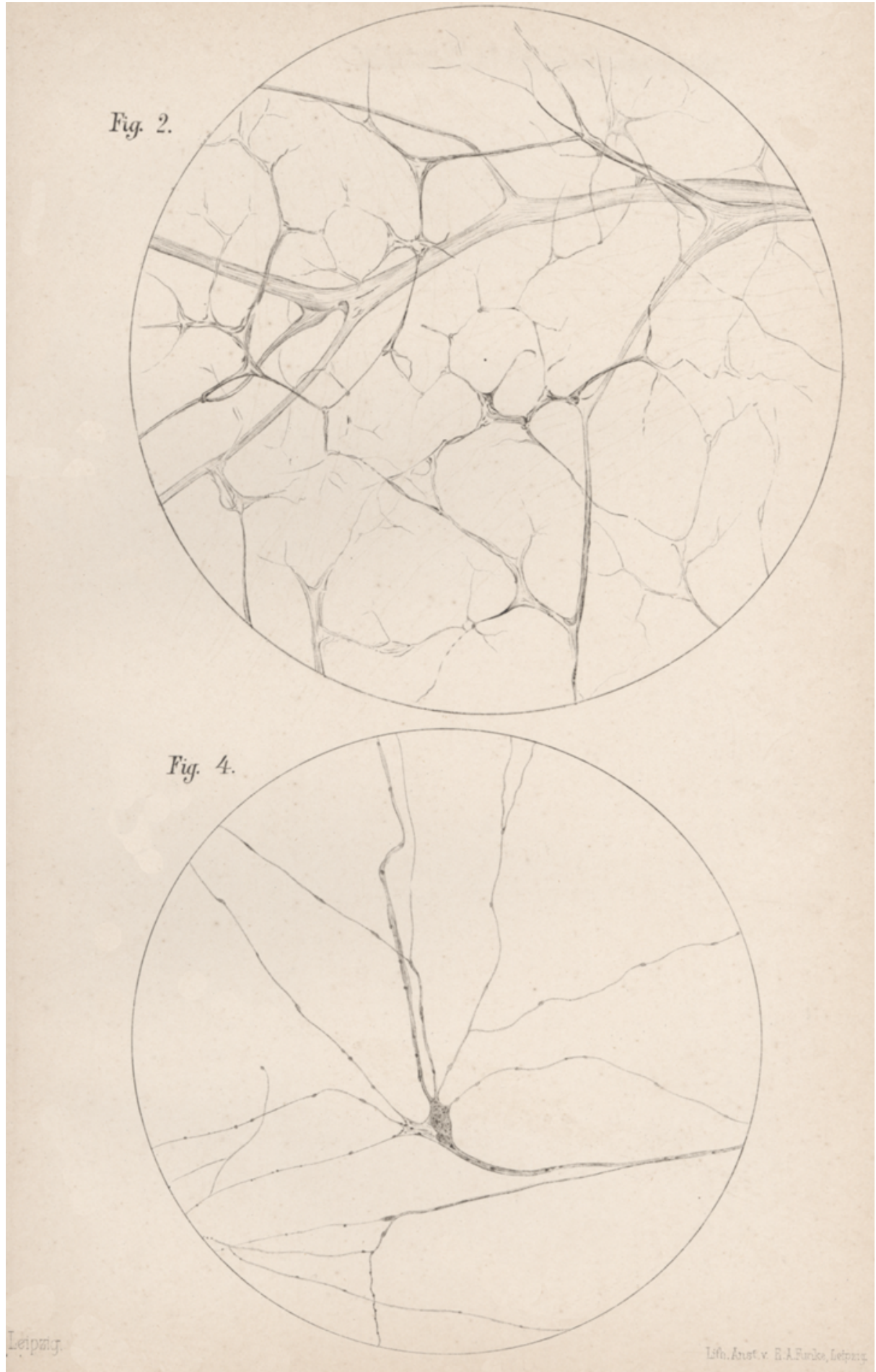




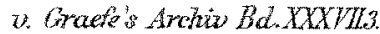

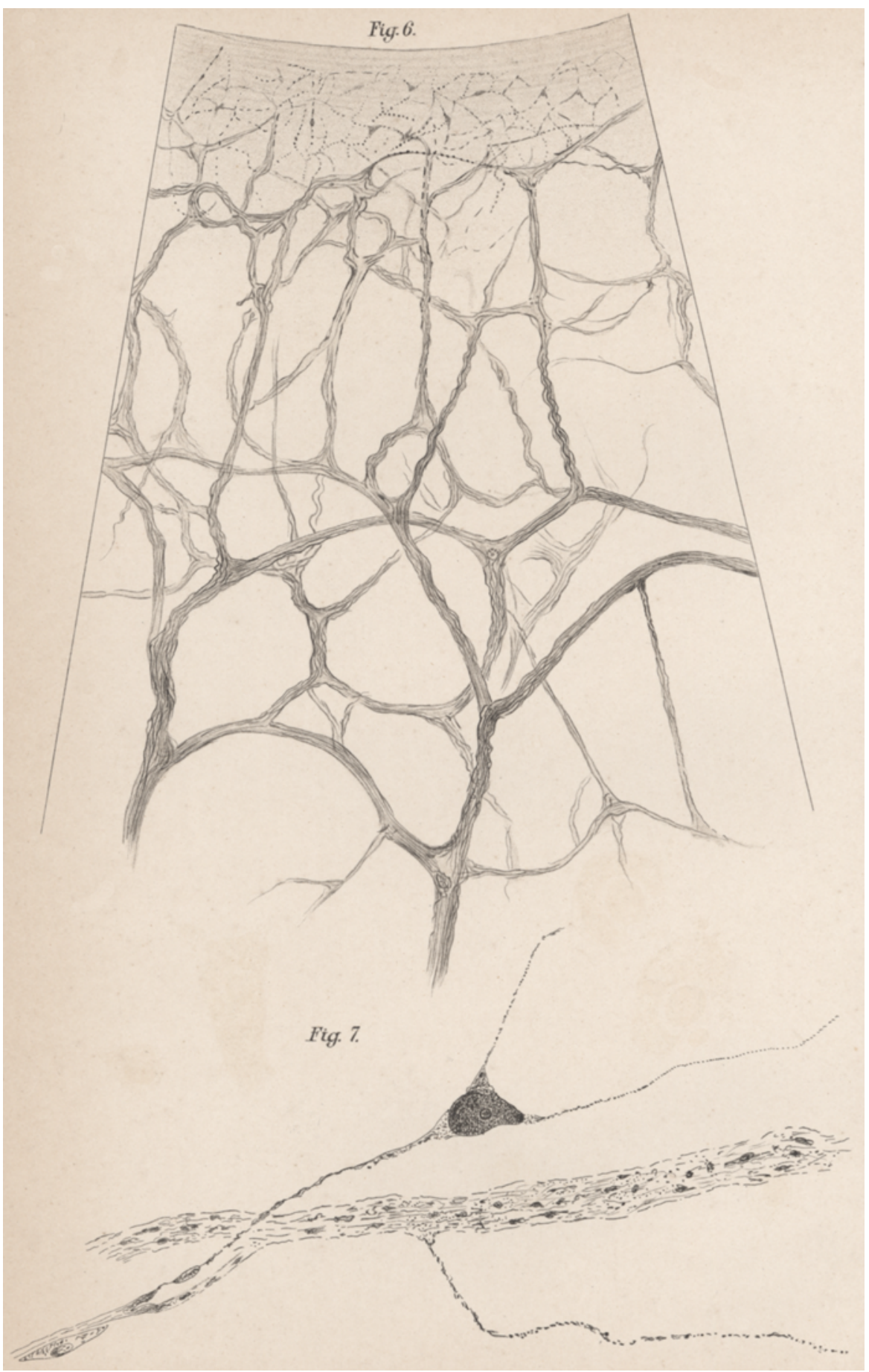


Taf.II.

Fig. 5.
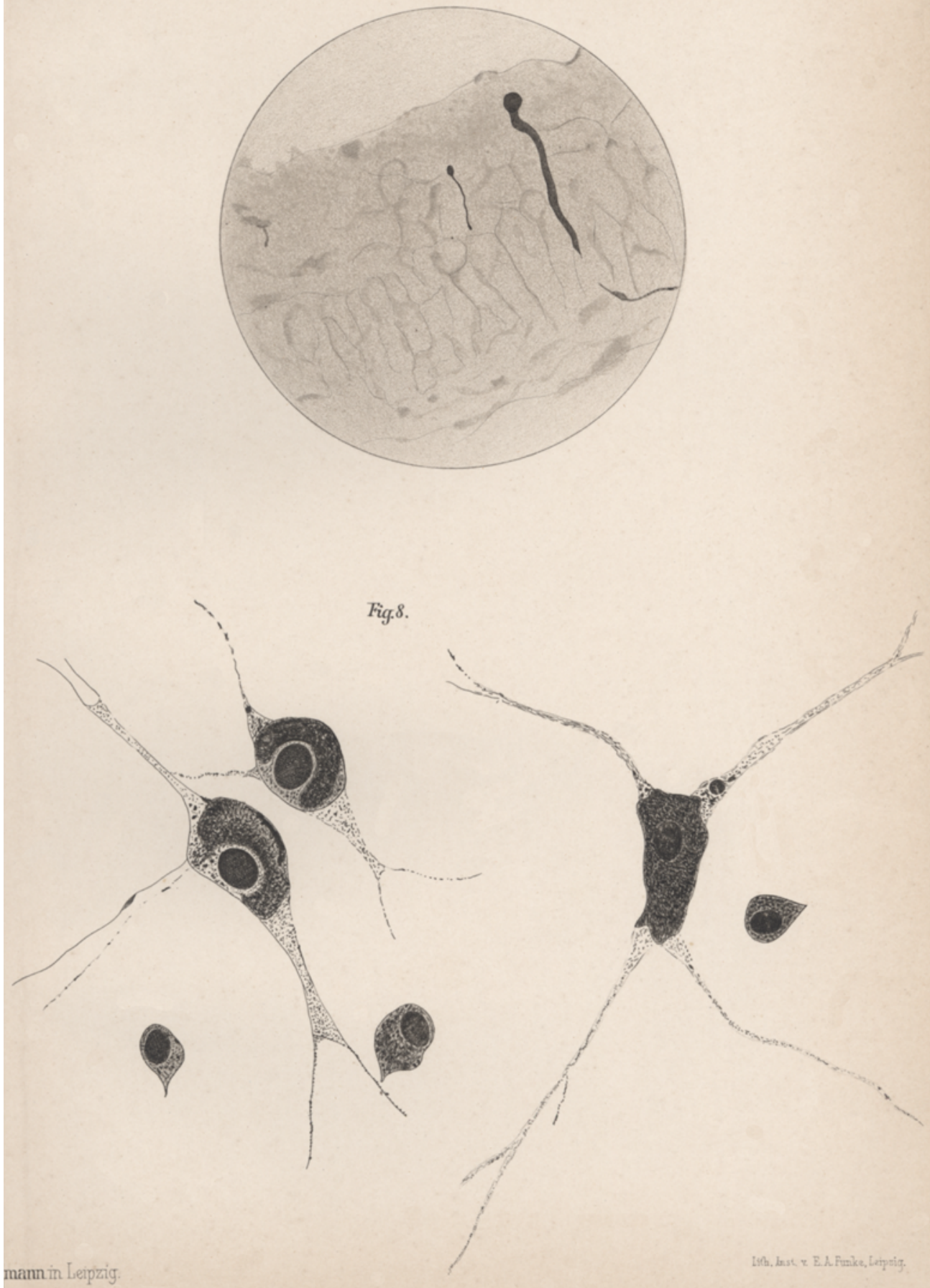